AZPH-TH-97/01

MPI-PhT 97-022

\title{
Does Conformal Quantum Field Theory Describe the Continuum Limits of 2D Spin Models with Continuous Symmetry?
}

\author{
Adrian Patrascioiu \\ Physics Department, University of Arizona, \\ Tucson, AZ 85721, U.S.A. \\ and \\ Erhard Seiler \\ Max-Planck-Institut für Physik \\ (Werner-Heisenberg-Institut) \\ Föhringer Ring 6, 80805 Munich, Germany
}

\begin{abstract}
It is generally taken for granted that two-dimensional critical phenomena can be fully classified by the well known two-dimensional (rational) conformal quantum field theories (CQFTs). In particular it is believed that in models with a continuous symmetry characterized by a Lie group $G$ the continuum theory enjoys an enhanced symmetry $G \times G$ due to the decoupling of right and left movers. In this letter we review the conventional arguments leading to this conclusion, point out two gaps and provide a conterexample. Nevertheless we justify in the end the conventional conclusions by additional arguments.
\end{abstract}

Since the works of Belavin, Polyakov and Zamolodchikov [1] as well as Friedan, Qiu and Shenker [2] it is generally accepted that two-dimensional critical 
phenomena can be fully classified by the well-known (rational) conformal Quantum Field Theories. This is also supposed to provide the explanation for the rational critical exponents occurring in these models.

In theories with a continuous symmetry group $G$ the conformal philosophy leads to a 'doubling' of the symmetry to $G \times G$ [3], with left and right chiral theories both separately invariant under $G$. Applying this philosophy to the model with the simplest continuous symmetry, namely the critical $O(2)$ model, it is concluded that it is completely describable by the Kac-Moody and Virasoro algebras associated to the massless free scalar field (see for instance [3]). This means in particular that the Noether current is a gradient, i.e.its curl vanishes.

In this letter we point out that the conventional arguments contain two gaps: firstly, it is not a priori guaranteed that the Noether current in the continuum exists as a local quantum field, and secondly, it could turn out that the Noether current is ultralocal in the Euclidean world, leading to its vanishing in the Minkowski world. For a more detailed discussion of the issues involved, see [4].

Conventionally, the arguments leading to the splitting of the theory into two independent 'chiral' theories and the ensuing enhancement of the symmetry are given in the framework of Minkowski space Quantum Field Theory. For our purpose here, let us first transcribe those arguments to the Euclidean setting: assuming a scale invariant continuum theory with a conserved current $j_{\mu}(x)$, Euclidean covariance requires that the two-point function $G_{\mu \nu}$ of $j_{\mu}$ is of the form

$$
G_{\mu \nu} \equiv\left\langle j_{\mu}(0) j_{\nu}(x)\right\rangle=\delta_{\mu \nu} \frac{b}{x^{2}}+\frac{a x_{\mu} x_{\nu}}{\left(x^{2}\right)^{2}} \quad(x \neq 0)
$$

and current conservation requires

$$
a=-2 b \text {. }
$$

This means that $G_{\mu \nu}$ is, up to a factor $b$, equal to the two point function of $\partial_{\mu} \phi$ where $\phi$ is the massless free scalar field (it is irrelevant here that the massless scalar field does not exist as a Wightman field). The two-point function of the dual current $\epsilon_{\mu \nu} j_{\nu}$ is then

$$
\tilde{G}_{\mu \nu} \equiv \epsilon_{\mu \lambda} \epsilon_{\rho \nu} G_{\lambda \rho}=G_{\mu \nu}
$$

so on the level of the two point function the dual current is also conserved. By general properties of local quantum field theory (Reeh-Schlieder theorem, 
see [5]) it follows that these conservation laws hold not only for the two point functions, but for the quantum fields $j_{\mu}$ and $\tilde{j}_{\mu}$ themselves. Conservation of the two currents $j$ and $\tilde{j}$ is equivalent to conservation of the two chiral currents $j_{ \pm}$.

So the two conservation laws together imply that

$$
j_{\mu}=\sqrt{b} \partial_{\mu} \phi
$$

where $\phi$ is the massless scalar free field, and also that

$$
j_{\mu}=\sqrt{b} \epsilon_{\mu \nu} \partial_{\nu} \psi
$$

where $\psi$ is another 'copy' of the massless scalar free field.

This argument is certainly correct, but it depends on the existence of the Noether currents as Wightman fields, and this is nontrivial and can actually fail, as the following example shows. This example is the two-component free field in $2 D$ in the massless limit, which also has a global $O(2)$ invariance. It is well known that the massless limit makes sense only for functions of the gradients of the fields. But the Noether current of the $O(2)$ symmetry cannot be written as a function of the gradients. It is also easy to see directly that its correlation functions do not have a limit as $m \rightarrow 0$ (see [四]).

The two-point function of the curl of the current of the free field is proportional to $1 /\left(x^{2}\right)^{2}$ in the massless limit, and if one tries to 'integrate' this to obtain the current two-point function, one obtains (imposing Euclidean covariance)

$$
G_{\mu \nu}(x)=-\delta_{\mu \nu} \frac{\ln x^{2}+\lambda}{8 x^{2}}+x_{\mu} x_{\nu} \frac{\ln x^{2}+1+\lambda}{4 x^{2}}
$$

with a free parameter $\lambda$. But this is not the Euclidean two-point function of a Wightman field, because it changes sign as we vary $x$ and therefore violates reflection positivity [6]. So here we have a case where both the curl and the divergence of the current exist as local fields (the divergence vanishes in Minkowski space), but the current itself does not. This situation is an instance of 'nontrivial local cohomology' [7, 8, 9]. So the first gap in the conventional argument is the assumption that the local cohomology is trivial.

We proceed now to show that in a continuum model with a critical point at $\beta_{c r t}<\infty$ with a continuous symmetry the situation of the counterexample ('nontrivial local cohomology') cannot arise. For the sake of definiteness we 
will concentrate on the $O(2)$ model, but it is straightforward to generalize the argument.

The $O(2)$ model is determined by its standard Hamiltonian (action)

$$
H=-\sum_{\langle i j\rangle} s(i) \cdot s(j)
$$

where the sum is over nearest neighbor pairs on a square lattice and the spins $s($.$) are unit vectors in the plane \mathbb{R}^{2}$. As usual Gibbs states are defined by using the Boltzmann factor $\exp (-\beta H)$ together with the standard a priori measure on the spins first in a finite volume, and then taking the thermodynamic limit.

The most interesting property of the model is its so-called KT transition [10], from a high temperature phase with exponential clustering to a low temperature one with only algebraic decay of correlations. A recent estimate for the transition point is 11

$$
\beta_{K T}=1.1197
$$

This number is not exact, but here all that matters is that the correlation length is so large that on the lattices we simulate it may be treated as infinite. The nature of the transition is supposed to be peculiar, with exponential instead of the usual power-like singularities, but this is not our concern here. Instead we want to study the model at its transition point. We are in particular interested in the correlations of the Noether current, given by

$$
j_{\mu}(i)=\beta \sin (\phi(i+\hat{\mu})-\phi(i))
$$

where

$$
s_{1}(i)=\cos (\phi(i)), s_{2}(i)=\sin (\phi(i))
$$

On a torus the current can be decomposed into 3 pieces, a longitudinal one, a transverse one and a constant (harmonic) piece. This decomposition is easiest in momentum space, and effected by the projections

$$
\begin{gathered}
P_{\mu \nu}^{T}=\left(\delta_{\mu \nu}-\frac{\left(e^{i p_{\mu}}-1\right)\left(e^{-i p_{\nu}}-1\right)}{\sum_{\alpha}\left(2-2 \cos p_{\alpha}\right)}\right)\left(1-\delta_{p 0}\right), \\
P_{\mu \nu=}^{L}=\frac{\left(e^{i p_{\mu}}-1\right)\left(e^{-i p_{\nu}}-1\right)}{\sum_{\alpha}\left(2-2 \cos p_{\alpha}\right)}\left(1-\delta_{p 0}\right)
\end{gathered}
$$


and

$$
P_{\mu \nu}^{h}=\delta_{\mu \nu} \delta_{p 0}
$$

with $p_{\mu}=2 \pi n_{\mu} / L, n_{\mu}=0,1,2, \ldots, L-1$.

We are mainly interested in the tranverse momentum space 2-point function

$$
F^{T}\left(p_{1}, 0\right)=\left\langle\left|\hat{j}_{2}\left(p_{1}, 0\right)\right|^{2}\right\rangle
$$

(for $p_{1} \neq 0$; the hat denotes the Fourier transform); the longitudinal twopoint function

$$
F^{L}\left(p_{1}, 0\right)=\left\langle\left|\hat{j}_{1}\left(p_{1}, 0\right)\right|^{2}\right\rangle
$$

is a constant because of current conservation. The constant is determined by a Ward identity to be $\beta E$ where $E=\langle s(0) \cdot s(\hat{\mu})\rangle$ (see 《4]).

The thermodynamic limit is obtained by sending $L \rightarrow \infty$ for fixed $p=$ $2 \pi n / L$, so that in the limit $p$ becomes a continuous variable ranging over the interval $[-\pi, \pi)$. The $O(2)$ model not only does not show spontaneous symmetry breaking according to the Mermin-Wagner theorem, but it has a unique infinite volume limit, as shown long ago by Bricmont, Fontaine and Landau [12]. The convergence to the thermodynamic limit is seen clearly in the Monte-Carlo simulations reported in 4 .

The continuum limit in the infinite volume is obtained as follows: let $\hat{F}(p ; \infty) \equiv \hat{T}(p)$ be the Fourier transform of the one-dimensional lattice function $T(n)$. In general $\hat{T}$ has to be considered as a distribution on $[-\pi, \pi)$, and it can be extended to a periodic distribution on the whole real line. The continuuum limit of $T(n)$ also has to be considered in the sense of distributions; it is obtained by introducing an integer $N$ as the unit of length, making the lattice spacing equal to $1 / N$. For an arbitrary test function $f$ (infinitely differentiable and of compact support) on the real axis we then have to consider the limit $N \rightarrow \infty$ of

$$
(T, f)_{N} \equiv \sum_{n} f\left(\frac{n}{N}\right) T(n)
$$

It is not hard to see that the right hand side of this is equal to

$$
\frac{1}{2 \pi} \int_{\infty}^{\infty} d q \hat{T}\left(\frac{q}{N}\right) \hat{f}(q)
$$

and converges as $N \rightarrow \infty$ to

$$
\frac{1}{2 \pi} \hat{T}(0) \int d q \hat{f}(q)=\frac{1}{2 \pi} f(0) \hat{T}(0)
$$


provided $\lim _{p \rightarrow 0} \hat{T}(p) \equiv \hat{T}(0)$ exists, expressing the fact that in this case the limit of $T$ is a pure contact term.

We want to prove rigorously that the continuum limit of the thermodynamic limits $\hat{F}^{T}(p, \infty)$ and $\hat{F}^{L}(p, \infty)$ of $\hat{F}^{T}(p, L)$ and $\hat{F}^{L}(p, L)$ are constants; the second fact is of course again just a restatement of the Ward identity (12), whereas the first one expresses the vanishing of curl $j$ in the continuum, thus confirming Affleck's claim regarding the enhancement of the continuous symmetry.

We use reflection positivity (RP) of the Gibbs measure formed with the standard action (see for instance [13]) on the periodic lattice. RP applied to the current two-point functions yields:

$$
F^{L}\left(x_{1}, L\right)=\sum_{x_{2}}\left\langle j_{1}\left(x_{1}, x_{2}\right) j_{1}(0,0)\right\rangle \leq 0
$$

for $x_{1} \neq 0$ and

$$
F^{T}\left(x_{1}, L\right)=\sum_{x_{2}}\left\langle j_{2}\left(x_{1}, x_{2}\right) j_{2}(0,0)\right\rangle \geq 0
$$

for all $x_{1}$. From these two equations it follows directly that

$$
0 \leq \hat{F}^{T}(p, L) \leq \hat{F}^{T}(0, L)=\hat{F}^{L}(0, L) \leq \hat{F}^{L}(p, L)=\beta E
$$

These inequalities remain of course true in the thermodynamic limit, but we have to be careful with the order of the limits. If we define $\hat{F}^{T}(p, \infty)$ and $\hat{F}^{L}(p, \infty)$ as the Fourier transforms of $\lim _{L \rightarrow \infty} F^{T}(x, L)$ and $\lim _{L \rightarrow \infty} F^{L}(x, L)$, respectively, one conclusion can be drawn immediately:

Proposition: $\hat{F}^{T}(p, \infty)$ and $\hat{F}^{L}(p, \infty)$ are continuous functions of $p \in$ $[-\pi, \pi)$.

The proof is straightforward, because due to the inequalities (19), (20) and (21) together with the finiteness of $\beta_{K T}$ the limiting functions $F^{L}$ and $F^{T}$ in $x$-space are absolutely summable.

But it is not assured that the limits $L \rightarrow \infty$ and $p \rightarrow 0$ can be interchanged, nor that the thermodynamic limit and Fourier transformation can be interchanged. On the contrary, the numerics presented in [4], as well as finite size scaling arguments (see below) suggest that

$$
\lim _{p \rightarrow 0} \lim _{L \rightarrow \infty} \hat{F}^{L}(p, L)>\lim _{L \rightarrow \infty} \hat{F}^{L}(0, L)
$$

and therefore also

$$
\lim _{p \rightarrow 0} \lim _{L \rightarrow \infty} \hat{F}^{L}(p, L)>\lim _{p \rightarrow 0} \lim _{L \rightarrow \infty} \hat{F}^{T}(p, L) .
$$


This fact plays an important role in closing the second gap in Affleck's arguments. But first we want to show the following:

Proposition: In the continuum limit both $\hat{F}^{L}(p, \infty)$ and $\hat{F}^{T}(p, \infty)(p \neq 0)$ converge to constants.

Proof: The proof is essentially contained in eqs.(17) and (18). We only have to notice that due to eq. (21) $\lim _{p \rightarrow 0} \hat{F}^{L}(p, \infty)$ and $\lim _{p \rightarrow 0} \hat{F}^{T}(p, \infty)$ exist.

In spite of this result, Affleck's claim could still fail in a different way if $\hat{F}^{T}(p, \infty)$ and $\hat{F}^{L}(p, \infty)$ converged to the same constant in the continuum limit. Let us denote the continuum limit of $\hat{F}^{T}(p, \infty)$ by $g$. Then the currentcurrent correlation in this limit is

$$
\left\langle j_{\mu} j_{\nu}\right\rangle \hat{(p)}=\beta E P_{\mu \nu}^{L}+g P_{\mu \nu}^{T}=g \delta_{\mu \nu}+(\beta E-g) \frac{p_{\mu} p_{\nu}}{p^{2}}
$$

So we see that if $g=\beta E$, the current-current correlation reduces to a pure contact term and vanishes in Minkowski space. Above we proved only that

$$
g \leq \beta E
$$

This gap in the conventional arguments will be closed using numerical simulation data together with finite size scaling arguments; we will show thereby that the continuum limit $g$ of $\hat{F}^{T}(p, \infty)$ is not equal to $\beta E$. For $\beta<\beta_{K T}$ the current two point function is decaying exponentially, hence its Fourier transform is continuous (and even real analytic). The same applies then to the longitudinal and transverse parts $\hat{F}^{L}(p, \infty)$ and $\hat{F}^{T}(p, \infty)$; in particular

$$
\hat{F}^{T}(0, \infty)=\hat{F}^{L}(0, \infty)=\beta E
$$

by the Ward identity mentioned above.

That does not, however, imply that at $\beta=\beta_{K T} \lim _{p \rightarrow 0} \hat{F}^{T}(p, \infty)=\beta E$, because the current two-point function cannot be expected to be absolutely summable there. On the contrary, if we can find that

$$
\lim _{L \rightarrow \infty} \hat{F}^{L}(0, L)<\beta E
$$

this implies also

$$
g=\lim _{p \rightarrow 0} \hat{F}^{T}(p, \infty)<\beta E
$$

because by eq. $(22) \hat{F}^{L}(p, \infty) \leq \lim _{L \rightarrow \infty} \hat{F}^{L}(0, L)$. 
The MC data taken at $\beta_{K T}$ and listed in Tab.1 indicate that

$$
d \equiv \beta E-\hat{F}^{L}(0, L)=\hat{F}^{L}\left(\frac{2 \pi}{L}, L\right)-\hat{F}^{L}(0, L)
$$

goes to a positive number $(<.68$ but probably $>.6)$, suggesting that indeed $g<\beta E$. But the question is whether this 'discontinuity' is a finite volume artefact or not. To address this issue we took data at $\beta<\beta_{K T}$ keeping the ratio $L / \xi$ fixed while increasing $\xi$. In this approach the massless continuum limit would correspond to $L / \xi \rightarrow 0$ (while $L / \xi \rightarrow \infty$ would correspond to the massive continuum limit in a thermodynamic box). Actually we use $L / \xi_{\text {eff }}$ instead of $L / \xi$ as an independent variable, where $\xi_{\text {eff }}$ is the effective correlation length measured on the lattice of size $L$; in the finite size scaling limit this is equivalent, because $L / \xi_{\text {eff }}$ becomes a unique monotonic function of $L / \xi$. The data listed in Tab.2 indicate that $d(L)=\beta E-\hat{F}^{L}(p, L)$ (the 'discontinuity' of $\hat{F}^{L}(p, L)$ at $\left.p=0\right)$ depends only on $L / \xi_{\text {eff }}$ in agreement with finite size scaling, and that it goes to a number above .6 in the massless continuum limit which is reached around $L / \xi_{\text {eff }} \approx 1.3$. The two sets of Monte-Carlo data together say that $\lim _{L \rightarrow \infty} d(L)$ is a number between .6 and .68 , and might actually be equal to $2 / \pi$. In any case, they provide convincing evidence that the Noether current is not an ultralocal field.

A.P is grateful to the Alexander von Humboldt Foundation for a Senior U.S.Scientist Award and to the Max-Planck-Institut for its hospitality; E.S. is grateful to the University of Arizona for its hospitality and financial support. Tab.1: The 'discontinuity' $d(L)=\beta E-g(L)$ at $\beta_{K T}$ for different values of $L$.

\begin{tabular}{r|r|r|r}
$L$ & $L / \xi_{\text {eff }}$ & $g(L)$ & $d(L)$ \\
\hline \hline 25 & 1.2575 & .09769 & $.7117(10)$ \\
50 & 1.2657 & .10666 & $.7027(18)$ \\
100 & 1.2667 & .11638 & $.6930(11)$ \\
200 & 1.2722 & .12357 & $.6858(08)$ \\
400 & 1.2839 & .12944 & $.6799(16)$
\end{tabular}

Tab.2: The 'discontinuity' $d(L)=\beta E-g(L)$ at various values of $\beta<\beta_{K T}$ and $L$. 


\begin{tabular}{r|r|r|r}
$\beta$ & $L$ & $L / \xi_{\text {eff }}$ & $d(L)$ \\
\hline \hline .93 & 12 & 1.8319 & $.3646(22)$ \\
.93 & 24 & 2.4441 & $.1926(24)$ \\
.93 & 36 & 3.2259 & $.0875(34)$ \\
\hline .96 & 18 & 1.8314 & $.3636(24)$ \\
.96 & 36 & 2.4242 & $.1929(31)$ \\
.96 & 54 & 3.1996 & $.0926(34)$ \\
\hline .99 & 32 & 1.8595 & $.3543(48)$ \\
.99 & 63 & 2.4537 & $.1919(64)$ \\
.99 & 64 & 2.4467 & $.1862(46)$ \\
.99 & 96 & 3.2110 & $.0909(43)$ \\
\hline 1.04 & 63 & 1.5868 & $.4709(59)$ \\
\hline 1.06 & 63 & 1.4549 & $.5411(49)$ \\
\hline 1.08 & 63 & 1.3722 & $.6012(36)$ \\
1.08 & 126 & 1.4188 & $.5818(46)$ \\
\hline 1.09 & 126 & 1.3990 & $.6174(18)$
\end{tabular}

\section{References}

[1] A.Belavin, A.Polyakov and A.Zamolodchikov, Nucl.Phys. B241 (1984) 333.

[2] D.Friedan, Z.Qiu and S.Shenker, Phys.Rev.Lett. 52 (1984) 1575.

[3] I.Affleck, Phys.Rev.Lett. 55, (1985) 1355.

[4] A.Patrascioiu and E.Seiler, Continuum Limit of 2D Spin Models with Continuous Symmetry and Conformal Quantum Field Theory, MPIPhT 97-xx, hep-lat/9703xxx.

[5] R.F.Streater and A.S.Wightman, PCT, Spin and Statistics and All That, Benjamin/Cummings, Reading, Mass., 1978.

[6] K.Osterwalder and R.Schrader, Comm. Math. Phys. 31 (1973) 83; Comm. Math. Phys. 42 (1975) 281.

[7] F.Strocchi, Phys.Rev 162 (1967) 1429.

[8] K.Pohlmeyer, Comm.Math.Phys. 25 (1972) 73. 
[9] J.Roberts in: Mathematical Problems in Theoretical Physics (G.Dell'Antonio, S.Doplicher and G.Jona-Lasinio, eds.), SpringerVerlag, Berlin 1978.

[10] J.M.Kosterlitz and D.J.Thouless, J. Phys. (Paris) 32 (1975) 581.

[11] M.Hasenbusch, K.Pinn and M.Marcu Physica A208 (1994) 124.

[12] J.Bricmont, J.R.Fontaine and L.J.Landau, Comm.Math.Phys. 56 (1977) 281.

[13] K.Osterwalder and E.Seiler, Ann.Phys. (N.Y.), 110 (1978) 440-471. 